\title{
Einleitung: Antike Mythologie in christlichen Kontexten der Spätantike ${ }^{1}$
}

In eine christliche Gefangenschaft sei der klassische Mythos während der Spätantike geraten. Zwar habe das Christentum ihn geduldet, doch: Solche Liberalität war die Schautoleranz der konsolidierten Macht. Mit so drastischen Worten beschreibt Hans Blumenberg in einem eindringlichen Aufsatz die Zäsur, die nach seiner Deutung die Christianisierung der Römischen Welt für die Geschichte des Mythos bedeutete. ${ }^{2}$ Die Zeit der Renaissance erscheint bei solchen Prämissen als Epoche der Befreiung für die alten Götter.

Diese Einschätzung gab der Philosoph an einem prominenten Ort, nämlich in dem 1971 erschienenen Sammelband Terror und Spiel, herausgegeben von Manfred Fuhrmann, dessen Ziel es laut Untertitel war, Probleme der Mythenrezeption zu behandeln. Er war ein Tagungsband der in Deutschland höchst einflussreichen Arbeitsgruppe Poetik und Hermeneutik, in der sich Vertreter verschiedener Fächer regelmäßig trafen, um ein zentrales Thema der Geisteswissenschaften unter interdisziplinären Gesichtspunkten zu beleuchten. $\mathrm{Zu}$ den Teilnehmern zählten neben Hans Blumenberg viele weitere bedeutende Gelehrte, etwa die Literaturwissenschaftler Wolfgang Iser und Hans Robert Jauß, der Religionswissenschaftler Jacob Taubes, der Historiker Reinhart Koselleck und aus den Altertumswissenschaften Forscher wie die Latinisten Manfred Fuhrmann und Reinhart Herzog oder der Althistoriker Christian Meier.

Die daraus resultierenden Sammelbände, gerade die älteren, sind zum Teil bis heute Standardwerke geblieben, und das zu Recht. ${ }^{3}$ Dies gilt auch für das genannte

1 Dieser Essay versucht den Ertrag des Sammelbandes zu profilieren; Literaturhinweise können nur exemplarisch sein und verstehen sich eher als Ergänzungen zu den umfänglichen Hinweisen in den Einzelbeiträgen des Bandes.

2 Hans Blumenberg, Wirklichkeitsbegriff und Wirkungspotential des Mythosbegriffs, in: Manfred Fuhrmann (Hg.), Terror und Spiel. Probleme der Mythenrezeption (Poetik und Hermeneutik 4), München $1990^{2}$, 11-66; s. die Diskussion der Teilnehmer dazu ibid., 527-547 und Hans Robert Jauß, Allegorese, Remythisierung und neuer Mythos. Bemerkungen zur christlichen Gefangenschaft der Mythologie im Mittelalter, ibid., 187-209. - Zu Blumenberg allgemein vgl. etwa Elizabeth Brient, The Immanence of the Infinite: Hans Blumenberg and the Threshold to Modernity, Washington, D.C. 2002; Franz Josef Wetz, Hans Blumenberg zur Einführung (Zur Einführung 289), Hamburg $2004^{2}$ (insbes. 100 -114 zum Mythos); Felix Heidenreich, Mensch und Moderne bei Hans Blumenberg, München 2005 (insbes. 67-74); Oliver Müller, Sorge um die Vernunft. Hans Blumenbergs phänomenologische Anthropologie, Paderborn 2005 (insbes. 177-181); Nadia Sels, The Function of Irony in Mythical Narratives. Hans Blumenberg and Homer's Ludicrous Gods, in: Wim M. J.van Binsbergen/Eric Venbrux (Hg.), New Perspectives on Myth. Proceedings of the Second Annual Conference of the International Association for Comparative Mythology, Ravenstein 19.-21. August 2008 (Papers in Intercultural Philosophy and Transcontinental Comparative Studies 5), Nimwegen 2010, 409-426.

3 Zu erwähnen wäre etwa Hans Robert Jauß (Hg.), Nachahmung und Illusion (Poetik und Hermeneutik 1), München 1964; Wolfgang Iser (Hg.), Immanente Ästhetik, ästhetische Reflexion: Lyrik als Paradigma 
Buch, das für die Behandlung der Mythenrezeption weiterhin unverzichtbar ist und zumal in den Literaturwissenschaften anregend wirkt. Daher wird dieser Band, der zugleich eine bestimmte Phase der Forschung eindrucksvoll repräsentiert, hier zum Ausgangspunkt der Einleitung genommen, obwohl er schon vor geraumer Zeit erschienen ist. ${ }^{4}$ Zudem erscheint die Auseinandersetzung mit dem plastischen Bild der christlichen Gefangenschaft des Mythos schon deswegen lohnend, weil es in seinen Vorannahmen so aufschlussreich ist. Denn der dogmatischen Ungebundenheit und Plastizität der Mythologie werden der dogmatische Ernst des Christentums und seine Unduldsamkeit gegenübergestellt. ${ }^{5}$

Blickt man auf die weiteren Beiträge jenes Bandes, so lassen sich zwei Subtexte beobachten: zum einen der eines krassen Gegensatzes zwischen Christentum und Heidentum, zum anderen der Versuch, dem Ganzen eine politische Dimension abzugewinnen, indem man eine moderne Begrifflichkeit, Ausdrücke wie Liberalität oder Toleranz, den antiken Gegebenheiten überstülpt. In beiden Punkten wird man heute vorsichtiger sein: Weder gelten die Entitäten Heidentum und Christentum als festgefügt noch wird man so unbefangen Begriffe der modernen politischen Kommunikation auf eine ältere Epoche übertragen, wie das in den siebziger Jahren geschah.

Charakteristisch für Blumenbergs Verständnis der Problematik erscheint folgende Passage: Die christliche Apologetik hat ihn (sc. den Mythos) ebenso mißverstanden, als sie - in absoluter Ernsthaftigkeit gegenüber den Widersprüchen der Mythologie und ihrer Frivolität - dort im Grunde den Konkurrenten sah, der wegen des vermeintlichen Gehaltes an ,Lehre، auszuschalten und dessen Stelle einzunehmen war, während doch in Wirklichkeit es nur die an den Gehalten der Mythologie praktizierte Liberalität gewesen ist, die als bleibend konkurrierende Unterströmung bis tief in die Renaissance und Neuzeit hinein dem Christentum als der Form des dogmatischen Ernstes und absoluter Verbindlichkeit den kontrastierenden Modus des Denkens und Sprechens darbot. Bezeichnend ist die empirisch schlicht unzutreffende Behauptung: Die Liberalität der

der Moderne (Poetik und Hermeneutik 2), München 1966; Hans Robert Jauß (Hg.), Die nicht mehr schönen Künste: Grenzphänomene des Ästhetischen (Poetik und Hermeneutik 3), München 1968.

4 Blumenberg hat diese Überlegungen später in sein großangelegtes Projekt der Arbeit am Mythos eingehen lassen, das das Thema in einer anthropologischen Dimension entfaltet, s. idem, Arbeit am Mythos, Frankfurt a. M. zuerst 1979; zur Einordnung dieser Arbeit etwa Ada Neschke-Hentschke, Rez. zu Hans Blumenberg: Arbeit am Mythos, Zeitschrift für philosophische Forschung 37 (1983), 448 - 453, die insbes. 450 den universalen Anspruch des Blumenberg'schen Mythosbegriffs kritisiert. Zur problematischen Entgegensetzung von Mythos und Dogma etwa Enno Rudolph, Mythos - Logos - Dogma. Eine Auseinandersetzung mit Hans Blumenberg, in: Oswald Bayer (Hg.), Mythos und Religion. Interdisziplinäre Aspekte, Stuttgart 1990, 58-79. Zur Kontextualisierung innerhalb der philosophischen Diskussionen zum Mythos Jörg Villwock, Mythos und Rhetorik. Zum inneren Zusammenhang zwischen Mythologie und Metaphorologie in der Philosophie Hans Blumenbergs, Philosophische Rundschau 32 (1985), 68-91.

5 Schon kritisiert von Jacob Taubes, Der dogmatische Mythos der Gnosis, in: Fuhrmann (s. Anm. 2), 145-156. Vgl. auch die Diskussion zu Blumenberg ibid., 527-547. 
Mythologie überlebte nur in den Übungsstücken der Rhetorik. ${ }^{6}$ Niemand wird heute daran zweifeln, dass das Überleben auf einer viel breiteren Grundlage erfolgte.

Bezogen auf die Spätantike, liegt verschiedenen Beiträgen jenes Bandes ferner eine Phasenmodell zugrunde, das vor allem ein kurz zuvor erschienener Aufsatz des Latinisten Manfred Fuhrmann entfaltet hatte: Fuhrmann vertrat die Auffassung, dass nach einer Zeit der Konfrontation im 5. Jahrhundert die christlichen und die heidnischen Eliten einen Kompromiss geschlossen hätten, der es erlaubt habe, bei Verzicht auf die traditionellen Kulte innerhalb bestimmter poetischer Gattungen die antike Mythologie zu tolerieren. ${ }^{7}$ Die Frage reduzierte sich also auf den gewiss wichtigen Aspekt der Elitenkonkurrenz, die den Mythos vor der Vernichtung bewahrte. Die Akzeptanz des Mythos wird dann zu einem Machterweis des Christentums. Fuhrmanns Blick richtete sich im Wesentlichen auf die lateinische Literatur und traf gar nicht das ganze Feld der außerliterarischen Präsenz von Mythologie. Und auch an jenen Debatten der Arbeitsgruppe Poetik und Hermeneutik nahm kein Archäologe teil, der Fuhrmanns Bild hätte korrigieren können; welche Bedeutung aber archäologische Quellen für diese Fragen haben, wird gerade im vorliegenden Band deutlich.

Es ist leicht, die Schwächen von Positionen zu markieren, die vor mehr als 40 Jahren formuliert wurden; darum geht es hier auch nicht. Ihre Wiedergabe soll vielmehr verdeutlichen, wie sich die Forschung der letzten Jahrzehnte entwickelt hat und dass dabei ein Thema erörtert wird, dessen Bedeutung über den Kreis der Spätantikespezialisten hinausreicht. Jeder Gang in ein Kunstmuseum führt eindrucksvoll die unbestreitbare Tatsache vor Augen, dass der Mythos in der christlichen Welt und auch in der säkularisierten kraftvoll überlebt hat. ${ }^{8}$ Doch auch außerhalb der bildungsbürgerlichen Provinz ist die Präsenz von Mythen bis in die Gegenwart hinein beachtlich. Um nur einige willkürlich zusammengegoogelte Beispiele zu nennen: Das sächsische Lößnitz rühmt

6 Blumenberg (s. Anm. 2), 20 und 21.

7 Manfred Fuhrmann, Die lateinische Literatur der Spätantike. Ein literarhistorischer Beitrag zum Kontinuitätsproblem, Antike \& Abendland 13 (1967), 56 - 79, 76f. sowie mit ähnlichen Formulierungen, aber bezogen auf die Mythen: idem, Die antiken Mythen im christlich-heidnischen Weltanschauungskampf der Spätantike, Antike \& Abendland 36 (1990), 138-151; vgl. idem, Rom und die Spätantike. Porträt einer Epoche, München u.a. 1994, 57. Zum Bild des Kompromisses s. Blumenberg (s. Anm. 4), 388; idem (s. Anm. 2).

8 Sehr vieles wird erschlossen bei Maria Moog-Grünewald (Hg.), Mythenrezeption. Die Antike Mythologie in Literatur, Musik und Kunst von den Anfängen bis zur Gegenwart (DNP Suppl.-Bd. 5), Stuttgart/Weimar 2008 (mit geschickt arrangierter Auswahlbibliographie); viele Hinweise in http:// www.oeaw.ac.at/kal/mythos/bibliomythos.pdf; reiches Material zur Präsenz von antiken Mythen in der Gegenwart in der Art, wie in den nächsten Sätzen geschildert, bei http://c2 m.free.fr/al/mytho.htm (abgerufen am 29.03.2014, 12:27). Udo Reinhardt, Der antike Mythos. Ein systematisches Handbuch (Rombach-Wissenschaften Paradeigmata 14), Freiburg/Br. 2011 vermittelt einen reichhaltigen, persönlich gefärbten Überblick, der knapp (409-413) auf christliche Kontexte eingeht; Hugo Rahner, Griechische Mythen in christlicher Deutung, Basel $1984^{4}$ will anders, als der Titel vielleicht suggeriert, keine systematische Darlegung bieten, sondern deutet das Verhältnis von Mythos und Christentum im Sinne einer christlichen Symboltheologie, die den Mythos - durchaus im Sinne mancher antiker Theologen - als Vorklang des Christentums auffasst. 
sich eines Gesundheits- und Fitnessclubs namens Apollo; ${ }^{9}$ Hercules ist eine beliebte Gestalt in Computerspielen, die sogar einen Helden-Parcours bieten, ${ }^{10}$ und auf ein Hotel namens Diana stößt man in fast jeder Stadt.

Nicht nur die antiken Mythologeme sind ubiquitär, auch der Begriff des Mythos ist beliebt - oder ist es das Wort? Ihm eignet jedenfalls eine proteushafte Vielfalt der Erscheinungsformen: Ganze Zeitalter und Denkweisen können als mythologisch bezeichnet werden; man findet Mythen in den verschiedensten Kulturen, in der Literatur wie auch im Alltag. Fast alles und fast jeder kann $\mathrm{zu}$ einem Mythos werden, ein Fußballverein wie ein Fernsehstar, so suggeriert es der Alltagsgebrauch des Wortes. Es kommt sogar zu interessanten Verschränkungen dreier verschiedener Wirklichkeitskonzepte, nämlich der Wissenschaft, des Wunderglaubens und der Mythologie, wie in der folgenden Zeitungsüberschrift: Forscher entlarven Hartz-IV-Wunder als Mythos. ${ }^{11}$ Mythos kann für etwas Bewundernswertes stehen, das eine gewisse historische Dauer hat, aber auch für das Falsche. Dem Christentum werden Mythen zugesprochen, doch kann es auch wie bei Hans Blumenberg als Welt des Dogmas und damit als Gegenentwurf zur mythologischen Weltsicht interpretiert werden.

Mehr Präzision fordert der wissenschaftliche Sprachgebrauch, doch hier lässt sich ebenfalls eine bemerkenswerte Spannbreite der Begriffsverwendung feststellen. Aleida und Jan Assmann grenzen angesichts der vielfältigen Bedeutungsschattierungen sieben Begriffe voneinander $a b$, die sie etwas technizistisch jeweils mit dem Kürzel $M$ und einer Ziffer versehen: ${ }^{12} M$ 1: Mythos als überwundenes Stadium kulturhistorischer Entwicklung; M 2: Mythos als zeitbedingte Einkleidung einer an sich zeitlosen Wahrheit, die etwa durch Allegorese erschlossen werden kann; M 3: Mythos als in weitester Auffassung sinnstiftende Erzählung; M 4: Mythen des Alltags, als Leitbilder, die kollektives Handeln und Erleben prägen; M 5: Mythos als narrativer Begriff, der im Sinne der aristotelischen Poetik Anfang, Mitte und Ende einer Erzählung betont; M 6: Literarischer Mythos, also Mythen, die ständig in der künstlerischen Verarbeitung umgeschrieben werden (hier wäre Entsprechendes für die bildende Kunst zu sagen, die aber nicht im Blick der Autoren ist); M 7: der Mythos im Sinne der großen Erzählungen, die einzelne formulieren; als ein Beispiel geben die Assmanns Friedrich Nietzsches Lehre von der ewigen Wiederkehr. Für die Beiträge des vorliegenden Bandes sind dabei insbesondere M 1-3 und M 6 bedeutsam, wenn man dieser

9 http://www.fitnessapollo.de, abgerufen am 06.02.2014, 12:24.

10 http://www.mogelpower.de/cheats/Hercules-_PC_488.html, abgerufen am 29.03.2014, 12:32.

11 Die Welt vom 03.02.2014 (http://www.welt.de/wirtschaft/article124489299/Forscher-entlarvenHartz-IV-Wunder-als-Mythos.html, abgerufen am 06.02. 2014 um 13:46).

12 Aleida u. Jan Assmann, Handbuch religionswissenschaftlicher Grundbegriffe 4 (1998), 179 - 200, s. v. Mythos, insbes. 179-181; s. ferner Walter Burkert/Axel Horstmann, Historisches Wörterbuch der Philosophie 6 (1984), 281-318, s.v. Mythos, Mythologie (ausführlicher: Axel Horstmann, Der Mythosbegriff vom frühen Christentum bis zur Gegenwart, Archiv für Begriffsgeschichte 23 [1979], 7-54; 197245); Fritz Graf u. a., DNP 8 (2000), 633 - 650, s.v. Mythos; Christine Schmitz/Francesco Zanella/Susanne Heydasch-Lehmann, RAC 25 (2013), 471-516, s.v. Mythos. 
Einteilung überhaupt folgen will. Arbeitspraktisch scheint es mir eher sinnvoll zu sein, eine relativ allgemeine Definition zu wählen, die aber unseren erfahrungsgesättigten Intuitionen entspricht: Mythen waren kollektiv bedeutsame, durch Tradition bekannte Erzählungen von Göttern und Heroen oft in ihrer Interaktion mit Menschen; auf jeden Fall haben es die Mythen der Antike, wie sie hier behandelt werden, mit personal gedachten Gestalten zu tun. ${ }^{13}$

Die Mythen sind allerdings nicht nur narrativ im Kontext der antiken Götterwelt zu sehen; sie gehörten auch in den Zusammenhang religiöser Kulte, die an bestimmten Orten über Jahrhunderte praktiziert wurden, auf der Grundlage oft sehr unterschiedlicher Varianten des Mythos, denen häufig ein aitiologischer Charakter zukam. Auf zahlreichen öffentlichen Kunstwerken, auch auf Münzen sah man mythische Bilder; für die lokale Identität gerade der Städte im Osten besaßen sie eine herausragende Bedeutung. ${ }^{14}$

Mythischen Motiven begegnete man so an verschiedenen sozialen Orten in unterschiedlicher Weise; die Allgegenwart der Mythen war gerade für die römische Kaiserzeit prägend: ${ }^{15}$ Man erblickte sie in Gestalt von Werken der bildenden Kunst auf öffentlichen Plätzen und in Privathäusern, man las über sie in der Literatur, man verfolgte sie namentlich bei den populären Pantomimen auf der Bühne und selbst in den mythischen Scharaden der Arena, man begegnete ihnen als einer literarischen Form der Philosophie, man zelebrierte ihre Tradition in Kulthandlungen. Unterschiede gab es sicherlich von Ort zu Ort: So hatte in Athen die Mythologie gewiss eine andere Sichtbarkeit und ein anderes Publikum als in Mainz bzw. Mogontiacum; auch literarische Milieus konnten je nach Region unterschiedlich sein.

Mythische Darstellungen besaßen offenbar durchaus auch Bedeutung für die Bewältigung der Kontingenz von Lebenskrisen: Als die Körperbestattung sich seit dem beginnenden 2. Jahrhundert wieder ausbreitete, wurden zahlreiche Sarkophage gefertigt. Hier sah man Mythen, die auf die Freuden und Pflichten des Lebens, aber auch und insbesondere auf den Tod beziehbar waren, mit so unterschiedlichen Motiven wie

\footnotetext{
13 Assmann/Assmann (s. Anm. 12) definieren aus religionswissenschaftlicher Perspektive den Mythos im allgemeinsten Sinne als den einer Gruppe vorgegebenen Fundus an Bildern und Geschichten (179). Doch scheint mit für die Behandlung speziell der Antike der personale Aspekt definitorisch wichtig zu sein.

14 Aus der reichen Literatur Tanja Scheer, Mythische Vorväter. Zur Bedeutung griechischer Heroenmythen im Selbstverständnis kleinasiatischer Städte (Münchener Arbeiten zur Alten Geschichte 7), München 1993; Ruth Lindner, Mythos und Identität. Studien zur Selbstdarstellung kleinasiatischer Städte in der römischen Kaiserzeit (Schriften der Wissenschaftlichen Gesellschaft an der JohannWolfgang-Goethe-Universität, Geisteswissenschaftliche Reihe 9), Stuttgart 1994; Bahadir Yildirim, Identities and Empire. Local Mythology and the Self-Representation of Aphrodisias, in: Barbara E. Borg (Hg.), Paideia. The World of the Second Sophistic (Millennium Studies 2), Berlin/New York 2008², $23-$ 52.

15 Paul Zanker/Björn Christian Ewald, Mit Mythen leben. Die Bilderwelt der römischen Sarkophage, München 2004, insbes. $37 \mathrm{f}$.
} 
Alkestis, Dionysos, Hippolytos oder den Niobiden. ${ }^{16}$ Insofern spricht einiges dafür, dass Mythen auch in nicht-christlichen Kontexten durchaus mit Fragen persönlicher Religiosität zu tun hatten.

Doch mit solchen Aussagen muss man vorsichtig sein. Was sah, las und hörte man denn, wenn es um Mythen ging? Wie wurden die Mythen perzipiert? Wem galten sie als religiös gehaltvoll? Maß man ihnen Bedeutung bei oder handelte es sich um eingeschliffene Floskeln und Ornamente, bei deren Verwendung man sich nichts dachte? Einfache Antworten darauf werden sich nicht finden, denn die Wahrnehmung der Mythen war gewiss kontextabhängig. Auch der Einzelne konnte einen Mythos je nach Situation ganz unterschiedlich wahrnehmen, sei es dass er über ihn beim Hören einer Persiflage lachte, dass er zu ihm aufblickte, wenn er ihn kunstvoll dargestellt fand, oder angesichts eines Sarkophags die Erinnerung an seine Sterblichkeit erfuhr. Im 3. Jahrhundert scheint sich in manchen Bereichen die Präsenz des Mythos verringert zu haben. Doch muss man dies keineswegs allein auf das Christentum zurückführen. ${ }^{17}$

Scheinbar liegt es auf der Hand, dass das Christentum kein unbefangenes Verhältnis zum Mythos haben konnte, wie auch immer er sich darstellte und wahrgenommen wurde. Er handelte von Göttern, in denen die Christen allenfalls Dämonen erblicken konnten, und er war in seinen wandelbaren Narrationen weit entfernt vom antiken Christentum, das sich in den ersten Jahrhunderten nach Christus zu einer Religion verbindlicher, verschriftlichter oder zumindest verbindlich fixierbarer Dogmen entwickeln sollte. Doch sollte man nicht vergessen, dass anfangs eine große Beweglichkeit und Vielfalt der Erzählungen über Jesus und über die Apostel bestand, wie bereits ein kurzer Blick in die apokryphe Tradition, aber eigentlich schon in die vier Evangelien lehrt. Gleichwohl äußerten Christen, zumal die Apologeten, fundamentale Kritik am Mythos, dessen Erzählungen in ihrer Widersprüchlichkeit und teils auch Obszönität sich nur allzu leicht lächerlich machen ließen.

Aber das Verhältnis von Christen zu Mythologischem lässt sich nicht über einen Leisten schlagen, zumal bereits von jüdischen Autoren verschiedene Formen des Umgangs mit dem Mythos erprobt worden waren. ${ }^{18}$ Denn zu jeder Zeit ist die Rede von „dem“ Christentum fragwürdig, da das antike Christentum nicht weniger vielfältig war

16 Dazu liegt schon eine reiche Literatur vor, s. nur etwa die mit einem reichen Repertorium versehene Synthese für die Stadt Rom Zanker/Ewald (s. Anm. 15).

17 S. für die Sarkophage, wo seit 220/30 die Zahl mythischer Darstellungen deutlich zurückgeht, Zanker/Ewald (s. Anm. 15), 255-263, ohne dass dies alle Bereiche der bildenden Kunst beträfe. Ibid., 262 ziehen sie die Möglichkeit eines christlichen Einflusses in Erwägung, erklären aber selbst, dass es dafür keinen Beleg gibt; zu Beginn des 3. Jahrhunderts wäre er kaum zu erwarten, da die Christen noch nicht in die Zentren der Gesellschaft vorgedrungen waren.

$18 \mathrm{Zu}$ Recht betont von René Bloch, Moses und der Mythos: Die Auseinandersetzung mit der griechischen Mythologie bei jüdisch-hellenistischen Autoren (Supplements to the Journal for the Study of Judaism 145), Leiden/Boston 2011. - Nicht behandelt werden kann hier die Rolle mythologischer Themen in jüdischen Bildwerken, wo sie seit dem 3. Jahrhundert immer wieder auftauchen, s. Lee I. Levine, Visual Judaism in Late Antiquity. Historical Contexts of Jewish Art, New Haven 2012, etwa 81f., $126-128,227,317-319,398,452$. 
als die Welt der Mythologie. In vielen der religiösen Äußerungen, die mit dem schwer zu definierenden Begriff der Gnosis bezeichnet werden und deren Anhänger sich zumindest in Teilen als christlich verstanden, spielten Mythen, die sich von den anderen antiken jedoch in Namensform und Erzählweise erheblich unterscheiden konnten, eine wie auch immer näher zu bestimmende, aber doch wesentliche Rolle. ${ }^{19}$ Gerade das könnte die Ablehnung, die dem Mythos bei anderen Christen entgegenschlug, befeuert haben. Für noch andere Christen wiederum, namentlich solche, die von der syrischen Kultur geprägt waren, waren die Mythen auch kulturell fremd. Im Gegensatz dazu wurden Christen, die ihre Sozialisierung in einer griechisch-römischen Umwelt erfahren hatten, mit Mythen groß; die Gebildeteren befassten sich im Unterricht intensiv mit ihnen. Bedeutsamkeit maßen allerdings wohl alle Christen dem Mythos bei, da er eben sichtbar für das religiös Andere, das Heidnische, stand. Auch das konnte ganz unterschiedlich aufgenommen werden: Während die einen Christen auf scharfe Konflikte mit dem setzten, was sie als heidnisch definierten, pflegten andere ein entspannteres Verhältnis zur Tradition.

Es ist daher bemerkenswert, dass im Prozess der Christianisierungen zwar die Kulte allmählich erloschen, die Mythen aber nicht untergingen. Eine Schlüsselepoche, die das Überleben der Mythologie möglich machte, bildete gewiss die Spätantike, mit der sich dieser Sammelband beschäftigt. Denn es war jene Epoche, in der das Christentum sich als Religion der Kaiser, der Eliten, der Gesellschaft insgesamt durchsetzte und schließlich alle Diskurse zu beherrschen schien. Und doch traf man in allen Phasen der Spätantike vielerorts auf mythologische Themen: Sie waren sichtbar bei öffentlich aufgestellten Kunstwerken und als Teil der Ausstattung privater Anwesen, lesbar in Texten, die dem Schulunterricht dienten, aber auch in solchen, die neu verfasst wurden, ausgedeutet in philosophischen Schriften, aber auch in theologischen Werken.

19 Zur Eigenart der Grundstruktur gnostischer Mythen s. Carsten Colpe, RAC 11 (1981), 537-659, s.v. Gnosis II (Gnostizismus), insbes. 635-639; ferner Walter Burkert, Kritiken, Rettungen und unterschwellige Lebendigkeit griechischer Mythen zur Zeit des frühen Christentums, in: Raban von Haehling (Hg.), Griechische Mythologie und frühes Christentum. Die antiken Götter und der eine Gott, Darmstadt 2005, 173-193, hier: 189-193. Hans Jonas spricht in seinem klassischen Werk (Gnosis und spätantiker Geist, Göttingen 1964ㄴ) von einer mythologischen Gnosis, der der erste, viel später erst ergänzte Band gewidmet ist. Akzentuiert wird die Rolle des Mythos von Taubes (s. Anm. 5); vgl. die Diskussion insbesondere zur Problematik des dort verwendeten Mythosbegriffs in: Fuhrmann (s. Anm. 2), 579 - 591. Die Diskussion ist weiter offen, zumal der Gnosisbegriff selbst sich als immer schwieriger erweist; vgl. Christoph Markschies, Die Gnosis, München 2010³ als pointierte Positionierung zugunsten der Verwendung des Begriffs der Gnosis Birger A. Pearson, Gnosticism and Christianity in Roman and Coptic Egypt, London u. a. 2004 (zur mythischen Dimension 205-207); ferner Antti Marjanen, ,Gnosticism‘, in: Susan Ashbrook Harvey/David G. Hunter (Hg.), The Oxford Handbook of Early Christian Studies, Oxford 2008, 203-220 (konziser Forschungsüberblick); einen theoretischen Neuansatz bietet Johann E. Hafner, Selbstdefinition des Christentums. Ein systemtheoretischer Zugang zur frühchristlichen Ausgrenzung der Gnosis, Freiburg u.a. 2003. 
Das christianisierte Reich konnte Mythologisches mithin zumindest ertragen. Unbestreitbar ist, dass sich für Mythen weiterhin sehr unterschiedliche Auffassungen anboten und dass auch Christen sie keineswegs einheitlich wahrnahmen. Als ein Beispiel sei auf die Ausstattung des Hippodroms von Konstantinopel mit mythologischen Kunstwerken durch Constantin den Großen hingewiesen, die BASSETT in diesem Band als Teil einer bewussten kaiserlichen Repräsentation interpretiert, mit der Constantin der Große die Ursprünge der Stadt in Erinnerung ruft und Sieghaftigkeit evoziert. Christliche Quellen machten sich darauf ihren je eigenen Reim: Während Euseb von Caesarea, der Bischof aus der Provinz, meint, dass durch die Aufstellung der Kunstwerke das Heidentum der Lächerlichkeit preisgegeben worden sei (Vita Constantini 3,54,2f.), sieht Sokrates, der hauptstädtische Kirchenhistoriker, in ihrer Verwendung im Hippodrom zwar eine Schwächung des Heidentums, doch auch einen Schmuck für die Stadt $(1,16,3)$ und ganz analog ist die Interpretation des Sozomenos, eines ebenfalls in der Hauptstadt wirkenden Kirchenhistorikers; auch bei ihm werden die Werke ästhetisiert (2,5,3f.).

Schon dieses Beispiel eines prominenten Ortes zeigt: Es kann nicht darum gehen, eine lineare Untergangs- oder Überlebensgeschichte der antiken Mythologie zu erzählen, sondern man hat es mit einer Vielfalt von Umgangsweisen, von Aneignungsprozessen zu tun, die in der spätantiken Gesellschaft möglich waren. ${ }^{20}$ Gerade die Pluralität, die Polyphonie der Spätantike hat die Forschung der letzten Jahrzehnte ja mit aller Deutlichkeit herausgearbeitet, und sie bestätigt sich auch in den Beiträgen dieses Bandes. Es wäre daher überaus spannend, eine Geschichte der Präsenz von Mythologie in Rom zu schreiben, die bewusst den Einschnitt der sogenannten Constantinischen Wende überbrücken würde. ${ }^{21}$

Das aber ist gegenwärtig nicht zu leisten. Dieser Sammelband zielt lediglich auf eine, indes überaus wichtige Phase der „Arbeit am Mythos“ in der mediterranen Tradition, eben auf die Phase der Christianisierung im spätantiken Reich, ${ }^{22}$ und es geht

20 Zum Begriff der Aneignung etwa Marian Füssel, Die Kunst der Schwachen. Zum Begriff der „Aneignung“ in der Geschichtswissenschaft, Sozial.Geschichte 21,3 (2006), 7-28 (Überblick zur Anwendung in der neueren Geschichte, vor allem bezogen auf Gegenstände); Dietmar Rothermund (Hg.), Aneignung und Selbstbehauptung. Antworten auf die europäische Expansion, München 1999, insbes. 4-9. Rémi Brague, Inclusion et digestion. Deux modèles d'appropriation culturelle, in: Philippe Capelle/Geneviève Hébert/Marie-Dominique Popelard (Hg.), Le Souci du passage. Hommage à Jean Greisch, Paris 2003, 77-96 = idem, Au moyen du moyen âge. Philosophies médiévales en chrétienté, judaïsme et islam, Paris 2006, 187-204, unterscheidet die beiden im Titel genannten Formen der Aneignung. Inclusion bewahre die Andersartigkeit des Gegenstandes, während die digestion den Gegenstand völlig verinnerliche, so dass er seine Unabhängigkeit verliere. Letzteres scheint Blumenberg zu unterstellen, doch dürfte inclusion die christliche Form der Aneignung des Mythos treffender beschreiben.

21 Anknüpfen könnte man in manchem an Jean Pépin, Mythe et allégorie. Les origines grecques et les contestations judéo-chrétiennes, Paris $1976^{2}$.

22 Für die frühere Geschichte des Christentums s. die Beiträge in Raban von Haehling (Hg.), Griechische Mythologie und frühes Christentum. Die antiken Götter und der eine Gott, Darmstadt 2005. Hier wird die Vielfalt der Aneignungsprozesse bereits sichtbar. 
um die griechisch-römische Mythologie in ihren vielfältigen Erscheinungsformen. Seine Beiträge untersuchen exemplarisch, wie der Mythos sich in christlichen Kontexten darbot. Damit stellt sich zugleich die Frage, warum, wo und wie der Mythos überleben konnte - und ob er dazu nur als Gefangener imstande war. Überdies ist zu erörtern, inwieweit sich unterschiedliche Phasen des Umgangs mit dem Mythos bestimmen lassen.

Hier versucht der vorliegende Band ein breiteres Panorama zu bieten. Vollständigkeit kann und will er dabei keineswegs beanspruchen - insbesondere fehlt der umfassende Bereich des Verhältnisses von Mythologie und Urbanistik fast vollständig. ${ }^{23}$ Nicht behandelt wird die Kleinkunst, etwa Öllampen ${ }^{24}$ oder Glasgerät ${ }^{25}$; auch die reichhaltigen Mosaiken werden hier nicht erörtert. Relativ wenig berücksichtigt sind ferner die Inschriften - um nur einige Beispiele für Lücken zu nennen. ${ }^{26}$ Es geht nicht darum, das Thema abzuschließen, sondern darum, den Forschungsstand zu verdeutlichen und weiterzuführen. Was in dem Band behandelt ist, vermittelt gleichwohl, so die Hoffnung, ein aufschlussreiches und anregendes Bild der Vielfalt im Umgang mit dem Mythos in der Spätantike.

Da die Verwendung von antiken Mythen in einem hohen Maße an Konventionen von Gattungen und Medien gebunden war, ist dieser Sammelband nicht nach mythologischen Motiven gegliedert und ordnet die Beiträge auch nicht chronologisch, sondern eben nach Medien und Gattungen, in denen Mythen vorkommen. Auf diese Weise können Verbindungen sichtbar werden, aber auch Ungleichzeitigkeiten zwischen Medien und Gattungen, die erklärungsbedürftig sind.

Das Epos und die Dichtung überhaupt haben in diesem Kontext seit jeher besonders große Aufmerksamkeit auf sich gezogen, da hier mythologische Elemente deutlich hervortraten, und zwar selbst wenn Autoren oder Adressaten in einen christlichen Zusammenhang gehören. Wie erwähnt, hat Fuhrmann die Entwicklung des Epos zum Ausgangspunkt der Geschichte des Mythos in der Spätantike genommen.

Mehrere Beiträge dieses Bandes befassen sich mit dieser Gattung: Am Beispiel des vieldiskutierten, um 400 aktiven Dichters Claudian zeigt CLAUDIA SCHINDLER auf, dass der Gebrauch der Mythen in der Poesie durchaus mit christlichen Vorstellungen bis hin

\footnotetext{
23 Etwa Angelos Chaniotis, Myths and Contexts in Aphrodisias, in: Ueli Dill/Christine Walde (Hg.), Antike Mythen. Medien, Transformationen und Konstruktionen, Berlin/New York 2009, 313-338.

24 John Lund, Motifs in Context. Christian Lamps, in: Jens Fleischer/Niels Hannestad/Marjatta Nielsen u.a. (Hg.), Late Antiquity. Art in Context = Acta Hyperborea 8 (2001), 199-214, der in verschiedenen Gattungen der materiellen Kultur Nordafrikas einen Übergang von heidnischen zu christlichen Motiven zu Beginn des 5. Jahrhunderts beobachtet; Arja Karivieri, Mythological Subjects on Late Roman Lamps and the Persistence of Classical Tradition, ibid., 179-198.

25 Jutta Dresken-Weiland, Bilder im Grab und ihre Bedeutung im Kontext der Christianisierung der frühchristlichen Welt, AnTard 19 (2011), 63-78, 73-78 zu Zwischengoldgläsern.

26 Wichtige Beobachtungen zur Verbindung von Inschrift und Kunstwerk bei Georgios Deligiannakis, Christian Attitudes towards Pagan Statuary: The Case of Anastasius of Rhodes, Byz 78 (2008), 142-158 (mit zahlreichen Literaturhinweisen), der betont, dass Kunstwerke Gegenstände ästhetischer Erfahrung und städtischen Stolzes blieben.
} 
zur Mythenkritik konform gehen kann. Ja, die Mythen werden in den entscheidenden, politischen Aspekten durch die Akteure der Jetztzeit überboten. So gesehen, kann Claudians Umgang mit dem Mythos, obwohl er in seiner Dichtung so prominent ist, nachgerade herabsetzend klingen. Zugleich bieten die Mythen namentlich der panegyrischen Dichtung einen Stoff, der aus spezifisch christlichen Texten nicht gewonnen werden konnte, da der Mythos breiter bekannt und anerkannt war.

Domenico ACCORINTI verdeutlicht am Beispiel des im 5. Jahrhundert wirkenden Epikers Nonnos, wie unterschiedlich das Nebeneinander von heidnisch qualifizierten Dionysiaka und christlicher Paraphrase des Johannes-Evangeliums ausgelegt werden konnte. Er selbst betont, dass das Verhältnis von beidem nicht als kontrastiv gesehen werden muss, dass vielmehr gewichtige Elemente beiden epischen Texten gemeinsam sind, vor allem das Motiv von Tod und Auferstehung, so dass ein Deutungsangebot entsteht, dank dem die heidnische und die christliche Tradition kompatibel werden, gerade weil die beiden Richtungen nicht als gegensätzlich erscheinen. Zugleich sieht AcCORINTI in den Dionysiaka einen Hinweis auf die Überlegenheit der christlichen Auferstehungslehre gegenüber heidnischen Vorstellungen zur Widererweckung von Toten, so dass im mythologischen Epos die christliche Tradition für die Leser, die in der Lage sind, die intertextuellen Bezüge wahrzunehmen, das Ältere überbieten würde.

UlRICH SCHMitzer behandelt den dichtenden Senator Sidonius Apollinaris, der um 470 zum Bischof von Clermont geweiht wurde. Er stellt fest, dass sowohl in seiner Panegyrik als auch in seiner Kleindichtung mythologische Motive eine stärkere Rolle spielen als in früheren Werken der Gattungen, und zwar auch dann, wenn diese wie ein kaiserlicher Panegyricus im öffentlichen Raum dargeboten wurden. Allerdings verloren sie ihre Brisanz. Schmitzer spricht von einer Einkapselung der paganen Gedankenwelt. Diese sei - hier zeigt sich eine Gemeinsamkeit mit ScHINDLERs Deutung indes weiterhin unverzichtbar gewesen, um etwa in der Panegyrik bestimmte Dinge zum Ausdruck zu bringen. Auch in der Kleindichtung bediente man sich daher gerne und ohne Scheu des Wissensspeichers, den die Mythen bildeten. Ihre Präsenz war eine poetische Option, kein religiöses Bekenntnis.

CHRISTIAN SchäFer untersucht einen Bereich, in dem Christentum und Heiden eine gemeinsame Grundlage hatten: platonisch inspirierte Denkweisen. Hier ist die Bewertung des Mythos oft gerade nicht etwas Trennendes; vielmehr ist beiden Traditionen gemeinsam, dass sie „wunderliche“ Elemente am Mythos feststellen: Die heidnische Mythenkritik wird vom Christentum aufgenommen, doch entwickelt sie sich auch weiter. Zumal am Beispiel des in der Zeit Julian Apostatas (361-363) wirkenden Sal(l)ustios wird erkennbar, wie die Deutung des Mythos sich angesichts der Herausforderung des Christentums entwickelt. Der Gedanke der (scheinbaren) Unpassendheit des Mythos, die auf Platon selbst zurückgeht, wird erneuert und der Mythos gerade den Gebildeten unter seinen Verächtern nahegebracht. Umgekehrt wirkt die platonische Mythendeutung auf die christliche Interpretationsweise biblischer Erzählungen zurück. Gerade auf diesem Feld tritt die Verschränkung christlicher und heidnischer Positionen bei der Arbeit am Mythos deutlich hervor: Weder steht das 
spätantike Heidentum hilflos-erstarrt da, noch sind die Christen gegenüber dem anderen verschlossen. Die Arbeit am Mythos schreitet lebendig fort.

Explizit christliche Texte kamen bei bestimmten Fragen auch nicht leicht am Mythos vorbei: WINRICH LöHR beleuchtet die Rolle, die Mythologeme bei christlichen Bischöfen der Spätantike spielen. Sie erscheinen nicht mehr als Gegenstand einer leidenschaftlichen Polemik: Zum einen gelten sie als Ausdruck einer überwundenen Phase, zum anderen als Bildungsgut, aber unter der Voraussetzung, dass sie neutralisiert sind, dass sie nicht als Figuren eines Glaubens verstanden werden. Damit konnten die Bischöfe indes, hier treffen die Überlegungen sich mit ScHÄFERs Ausführungen, an heidnische Auffassungen anschließen, da die mythischen Gestalten für viele gerade nicht die religiöse Bedeutung hatten, die Christen jenen der Bibel beimaßen. Die entscheidende Frage war vielmehr, inwieweit sie zur Sittlichkeit ermunterten. Die mythologischen Themen konnten als Ausdruck des gemeinsamen Codes der Gebildeten Akzeptanz finden, indem man zugleich die Überlegenheit der christlichen Lehre betonte: Christentum und Heidentum wurden in dieser Deutung durch curriculare Hierarchisierung integriert, womit die Mythen letztlich ihren heidnischen Charakter im Sinne eines unauflöslichen Gegensatzes zum Christentum einbüßten und den christlichen Lehren gewissermaßen vorgeschaltet waren.

An Erich Auerbachs Literaturtheorie anknüpfend fragt HENRIKE ZILLING danach, inwieweit die christlichen Helden auf heidnische Heroen referieren. Dafür verweist sie auf Motivähnlichkeiten zwischen antiken Heroen und den christlichen der Evangelien, aber besonders auch der Märtyrerberichte. Die Geschichte des frühen Christentums wird so $\mathrm{zu}$ einer Geschichte von Helden, die figural mit den klassischen Heroen verbunden sind, wobei zugleich Jesus Christus so viele Besonderheiten aufweist, dass er trotz aller Gemeinsamkeiten mit einem klassischen Heros eine Gestalt sui generis bleibt. Besonders deutlich werden die Gemeinsamkeiten im Vergleich zwischen Herakles und dem Märtyrerbischof Polykarp. Mythos und Martyrium erscheinen hier in einer Kontinuität. Ferner können Mythen dazu dienen, Verhaltensweisen von Christen zu begründen. In dieser Deutung werden Teile des Mythos somit von Christen gleichsam vereinnahmt.

Herakles spielt noch im 7. Jahrhundert eine bedeutende Rolle, wie MischA MEIER zeigt. Der Rekurs auf Herakles und seine Leistungen erlaubt es dem Kaiser Herakleios (610-641), sich an Christus anzunähern. Hier sind Mythos und Christentum nicht nur koexistent, sondern sie verstärken sich gegenseitig. Relativ subtile Anspielungen genügten in der Zeit, als das Reich vollständig christianisiert erscheint, um den Mythos zu evozieren. So wird Herakles erneut zu einem wichtigen Element der kaiserlichen Selbstdarstellung, wenngleich in ganz andere Manier als unter Commodus und den Tetrarchen. Die fortwährende Adaptabilität des Mythos - MEIER spricht sogar von einer „Herakles-Mode“ - wird hier überaus deutlich.

Einer weiteren mythischen Gestalt widmet sich FABIEnNE JoURdan, indem sie die Aneignung des Orpheus-Mythos durch Christen untersucht, die sehr unterschiedlich erfolgen konnte: Dieser Mythos hatte eine besondere Bedeutung, da Orpheus von vielen Christen als Begründer der griechischen Religion insgesamt aufge- 
fasst wurde. Er konnte einerseits deswegen denunziert werden, doch wird ihm auch eine Hinwendung zum Monotheismus zugeschrieben, so dass er auch als Vorbild für Konversion zu dienen vermag. Doch selbst wenn Orpheus positiv gesehen wurde, näherten die Christen sich ihm im Modus der Überbietung, da eben die Hinwendung zum Christentum der entscheidende Schritt war.

Clifford ANDo widmet sich den Mythen der Vor- und Frühgeschichte Roms, die in der Spätantike wohlbekannt waren. Jetzt aber wurden sie angesichts der Herausforderung durch das jüdisch-christliche Narrativ im Sinne einer Geschichte verstanden, die historischer Kohärenz bedurfte. Bei den Kommentatoren zur frühen römischen Geschichte ist daher ein Streben nach Systematisierung feststellbar, das einem Plutarch etwa fehlte. Insofern verwandeln diese Autoren Mythos in eine Narration nach Art der Historiographie. Der Mythos erweist sich weiterhin als veränderlich und kann so ohne weiteres in christlichen wie nicht-christlichen Kontexten weiterleben.

Durch eine Behandlung literarischer wie archäologischer Quellen verdeutlicht JöRG RÜPKE die Möglichkeit eines Nebeneinanders von Christlichem und Heidnischem in ganz verschiedenen Medien, womit die binäre Gegenüberstellung von beidem im Sinne eines Weltanschauungskampfes, wie man in der Zeit der Ideologien (Karl Dieter Bracher) gerne sagte, in Frage gestellt wird. RüPKE wählt den Kalender von 354 als ein Zeugnis einer senatorischen Haltung aus, die über den üblichen Konflikten steht. Vor diesem Hintergrund wird auch die Präsenz mythologischer Motive in den Katakomben an der Via Latina, die aus der gleichen Zeit stammen, verständlich. Überhaupt scheinen die Akteure dieses Milieus das Verhältnis von Heidentum und Christentum nicht primär als konflikthaft gesehen und mythische Themen nicht notwendig religiös interpretiert zu haben.

SARAH BASSETT erörtert den Umgang mit der klassischen Kunst im spätantiken Konstantinopel, dem Neuen Rom. Sie betont die anhaltende Präsenz von Statuen mit mythologischen Themen. In Konstantinopel wurden Statuen sogar in einem bestimmten System gezielt an öffentlichen Plätzen aufgestellt. Vor allem evozierten sie die mythische Tradition über Konstantinopels Ursprünge in Rom und damit indirekt in Troja. Im 5. Jahrhundert, als die Zahl der kaiserlichen Portraits wuchs, wurden mythologische Ensembles verstärkt in privaten Zusammenhängen präsentiert. Hier beschwor man nicht mehr die mythische Tradition der Stadt herauf, vielmehr rief man die frühere religiöse Bedeutung vieler Statuen sowie ihren kunsthistorischen Rang in Erinnerung; doch wurden sie auch zusammen mit Gestalten von wilden Lebewesen präsentiert, damit exotisiert, vielleicht auch gedemütigt, wobei zugleich auch ihr ästhetischer Rang in Erscheinung treten konnte. Von apologetischer Verdammung entwickelte sich die christliche Haltung gegenüber mythologischen Statuen zu institutionalisierter Akzeptanz.

Eine selten genutzte Quelle behandelt Troels MYRuP KRISTENSEN: ägyptische Textilien, die man in Gräbern gefunden hat. Diese Objekte haben den Vorzug, dass sie nicht in die Welt der Eliten gehören - KRISTENSEN spricht bei den Nutzern vorsichtig von middle classes - und eine in großen Mengen produzierte Ware darstellen, ähnlich wie etwa Öllampen. Auch bei den Textilien wird ein Nebeneinander klassischer, nicht 
spezifisch ägyptischer mythologischer und christlicher Motive sichtbar; auch hier ist davon auszugehen, dass der Mythos nicht als unchristlich definiert, sondern als ein Teil der eigenen Kultur aufgefasst wurde, den die Aneignung christlicher Motive nicht zwingend affizierte. Denn diese Textilien wurden nicht nur als schöne Objekte gesehen, sondern als Element der Selbstdarstellung im Tode, wobei die Verwendung eines mythischen Motivs keineswegs zwingend bedeutet, dass der Besitzer damit über seine religiöse Identität Auskunft geben wollte. Das bedeutet zugleich, dass die Zuordnung von Gräbern zum Christentum und Heidentum nicht möglich ist. Gemeinsame kulturelle Prägungen blieben von der Definition des Mythos als heidnisch, die manche Theologen propagierten, unberührt.

Die Beiträge des Sammelbandes machen, so fragmentarisch das Bild noch ist, verschiedenes deutlich: Aufgelöst hat sich das Paradigma einer Mythologie, die zwar Plastizität besaß, aber letztlich selbstreferentiell war. Vielmehr wird die Mythologie in einer Vielfalt von Verwendungsweisen gesehen, im modernen Universitätsjargon gesprochen: in ihrer Anschlussfähigkeit. Es kommt darauf an, wo, wann und wem Mythologisches begegnet. Daraus ergeben sich vielfältige Möglichkeiten der Aneignung. Es gibt daher auch keine lineare Entwicklung des Niedergangs oder Bedeutungsverlusts des Mythos, sondern verschiedene Modi der Rekontextualisierung.

Dabei ist auch ein quellenkritisches Problem zu berücksichtigen: Die Überlieferungsgeschichte führte dazu, dass literarische Quellen bewusst selektiert wurden, und zwar im Sinne christlicher Vorstellungen, die auf eine Abgrenzung vom Heidentum setzten - auch wenn manches andere überlebt hat. Wenn man sich in einem Gedankenexperiment vorstellt, dass nur die archäologische Überlieferung erhalten geblieben wäre, so hätte man weitaus stärker den Eindruck eines Nebeneinanders von Christlichem und Heidnischem - wenn man überhaupt diese Kategorien als das zentrale Interpretament wählen würde. ${ }^{27}$ Man würde zudem feststellen müssen, dass auch noch mindestens bis zum ausgehenden 4. Jahrhundert Kunstwerke mit mythologischem Gehalt produziert wurden. ${ }^{28}$

Die in der bildenden Kunst besonders sichtbare Verbindbarkeit von mythischen und christlichen Themen ist auch anderswo greifbar. Was sind die Gründe für das Überleben? Hier lassen sich nur einige vorläufige Überlegungen anstellen: Für die Bewohner antiker Städte, die weiterhin fortwährend auf Kunstwerke der klassischen Tradition trafen, mochte die Koexistenz von Mythischem und Christlichem einfach ein selbstverständlicher Teil ihrer Lebenswelt gewesen sein. Derartige Dispositionen

27 Die relative Schnelligkeit des Übergangs für die funerären Zusammenhänge betont Dresken-Weiland (s. Anm. 25), wobei es im 3. Jahrhundert auch zu einem pragmatischen Nebeneinander kam: Anscheinend wurden auf Sarkophage mit herkömmlichen Motiven Deckel mit Jonas-Darstellungen gelegt.

28 Marianne Bergmann, Chiragan, Aphrodisias, Konstantinopel. Zur mythologischen Skulptur der Spätantike (Palilia 7), Wiesbaden 1999; Christiane Vorster, Spätantike Bildhauerwerkstätten in Rom. Beobachtungen zur Idealskulptur in der nachkonstantinischen Zeit, JDAI 127/8 (2012/3), 393-498. 
tauchen aber in Schriftquellen typischerweise nicht auf, da sie eben nicht reflektiert wurden.

Ferner bedurfte gerade eine Gesellschaft, die sich religiös so radikal wandelte wie die spätantike, vermutlich neutraler Räume, um eine inklusive Kommunikation zu ermöglichen und damit den Zusammenhalt zumal der religiös so diversen Eliten zu bewahren: ${ }^{29}$ Über bestimmte Dinge, und das betrifft sowohl die politische Ordnung als auch die Selbstdarstellung von Eliten, ließ sich offenbar besser sprechen, wenn man traditionelle, weithin bekannte und akzeptierte Themen wählte, die auch wenn sie mythologisch waren, nicht als religiös distinktiv begriffen werden mussten.

Möglicherweise wäre noch eine weitere Voraussetzung dafür zu berücksichtigen, dass der klassische Mythos die Christianisierungen in verschiedenen Bereichen der Gesellschaft überleben konnte: In der Antike entwickelte man, wie ScHÄFER verdeutlicht, früh ein reflexives Verhältnis zu Mythen, nahm sie also nicht einfach hin, sondern durchdachte sie, überformte sie auch, spielte sogar mit ihnen, so dass sie leicht vom religiösen Kontext ablösbar waren. Noch stärker als für Griechenland gilt dies für Rom, wohin viele griechische Mythen transponiert worden waren, so dass sie ihren örtlichen Bezug verloren, teils auch einen neuen fanden. Das bewirkte auch die von Blumenberg beschworene hohe Plastizität des Mythos, seine Ungeeignetheit zur Markierung von Ketzern und Apostaten, die dazu führte, dass man einen falschen Gebrauch dieser Erzählungen schwerlich zu denunzieren vermochte - und da konnten am Ende auch Christen sich seiner bedienen. ${ }^{30}$

Ferner wird darüber nachzudenken sein, wie hilfreich die binäre Unterscheidung zwischen Heidentum und Christentum überhaupt ist, die eine einseitige Perspektive vermittelt, gerade weil sie von manchen theologischen Texten der Antike favorisiert wurde. Denn es gab christliche Gruppen, die auf Abgrenzung setzten und die in der Überlieferung sehr präsent sind. Doch bildeten sich auch andere Gruppen: Einiges spricht dafür, dass für manche Christen gerade der Eliten das, was andere Christen als heidnisch verwarfen, als Altbekanntes erträglich war, während Heiden Christliches, also zunächst einmal Neuartiges, nur schwer akzeptieren konnten.

Offenbar war der Mythos auch religionsübergreifend wichtig für die Konstitution der Eliten, insofern als Mythenkenntnis ein Mittel der Statusdistinktion bildete. Es gab starke Gruppen von konsensorientierten Akteuren, von center-Christians und center-pagans, wie sie kürzlich genannt wurden. ${ }^{31}$ Die antike paideia, der kein spezifisch christliches Schulwesen gegenüberstand, vermittelte den Gebildeten jenseits aller sonstigen Differenzen einen gemeinsamen Code, der besonders in der Dichtung sichtbar wurde und für den Mythisches unverzichtbar war - das Bildungswesen hatte ja nie der religiösen Unterweisung gedient. Insofern bedeutet die religiöse Neutralisierung des Mythos hier den Normalzustand. Julian sollte mit seinen religiös getönten

29 Hartmut Leppin, Christianisierungen im Römischen Reich: Überlegungen zum Begriff und zur Phasenbildung, ZAC 16 (2012), 245-276, 259-265.

30 Blumenberg (s. Anm. 2), 17.

31 Alan Cameron, The Last Pagans of Rome, Oxford 2011. 
Forderungen an die Rhetoren, dass sie hinter den Inhalten der von ihnen unterrichteten Texte zu stehen hätten, ein isolierter Einzelfall bleiben. ${ }^{32}$

Mir scheint es aufgrund des Gesagten hilfreich, heuristisch nach mindestens drei Formen des christlichen Umgangs mit Mythen zu differenzieren, die sich übrigens nicht wechselseitig ausschließen: Gerade die frühen christlichen Autoren stellen den falschen Mythos der christlichen Wahrheit entgegen. Mythos wurde hier nachgerade zu einem Gegenstand der Polemik und Abgrenzung. Das konnte geschehen, indem man, an die hergebrachte Mythenkritik seit Xenophanes von Kolophon und Platon anknüpfend, Mythen als kindisch, widersprüchlich oder frivol abtat, aber auch in euhemeristischer Weise als Überhöhung historischer Geschehnisse entlarvte, um zu bekräftigen, dass allein der christliche Gott Verehrung verdiente. Diese Haltung löste da, wo Christen Gewalt anwandten, bisweilen Handlungen aus, die das mythologische Werk depotenzierten: über die Kennzeichnung von Kunstwerken mit einem Kreuz ${ }^{33}$ bis hin zu deren physischer Vernichtung und in Einzelfällen zu Versuchen der Eliminierung von Mythen aus dem Bildungsbetrieb. Gerade diese Form der bisweilen durchaus auch physischen Arbeit am Mythos belegt indes, wie ernst die Kraft des Mythos von einigen genommen wurde. Doch verloren diese zerstörerischen Formen des Umgangs, wie es scheint, in der Spätantike an Bedeutung.

Eine andere Form des christlichen Umgangs mit Mythen war ihre Neutralisierung in religiöser Hinsicht. Indem man - und dieses „man“ schließt auch Bischöfe ein (LöHR) - sie als kulturell notwendiges, wenngleich der christlichen Botschaft unterlegenes Bildungswissen einordnete oder als ästhetische Phänomene betrachtete, gar musealisierte (BASSETT), erleichterte man ihre Integration in eine christliche Welt; die Allegorese, die sich schon unter heidnischen Philosophen und Mythographen entwickelt hatte, war dazu ein probates Mittel. ${ }^{34}$ Ein plastisches Bild wählt SCHMITZER, laut dem eine Einkapselung nötig war, um die Mythologie ihrer Brisanz zu berauben. Umgekehrt sah ein bekennender Heide wie Kaiser Julian sofort die Gefahr, die für seine religiöse Position aus einer solchen Haltung zum Mythos folgte und ereiferte sich über Christen, die ohne religiöse Anteilnahme klassische Texte lehrten. ${ }^{35}$

Eine dritte Form, die sich mit der zweiten überlappt, wäre die der Aneignung von Mythen, indem man deutlich machte, wie überlegen das Christentum gegenüber der Mythologie war, die so eine Stufe zum wahren Verständnis des Christentums bilden

32 Grundlegend aufgrund der Einbeziehung seiner umfänglichen älteren Forschungen Alan Cameron, Poetry and Literary Culture in Late Antiquity, in: Simon Swain/Mark Edwards (Hg.), Approaching Late Antiquity. The Transformation from Early to Late Empire, Oxford 2004, 327-354. Zu Julians sog. Rhetorenedikt s. Cod. Theod. 13,13,5 mit Jul., Ep. 61c (Bid.) und die völlige Ablehnung durch den differenziert über den Kaiser urteilenden Ammianus Marcellinus 22,10,7; 25,4,20.

33 Aber auch mit dieser Kennzeichnung konnten sich unterschiedliche Deutungen verbinden, s. Troels Myrup Kristensen, Miraculous Bodies. Christian Viewers and the Transformation of ,Pagan`Sculpture in Late Antiquity, in: Stine Birk/Birte Poulsen (Hg.), Patrons and Viewers in Late Antiquity (Aarhus Studies in Mediterranean Antiquity 10), Aarhus 2012, 31-66.

34 Grundlegend Pépin (s. Anm. 21).

35 Jul., Ep. 61c (Bid.). 
konnte - in diesem Sinne deutet im vorliegenden Band ACCORINTI die Dionysiaka des Nonnos, aber auch ScHINDLER Claudian und BASSETT den Umgang mit bildender Kunst. Oder aber man deutete, auch hier bot sich die Methode der Allegorese an, etwas vordergründig Unwahres wie die mythischen Erzählungen als verhüllte Wahrheit und bezog so Mythen auf das Christentum. Die Aneignung wurde dadurch erleichtert, dass viele Mythen christlichen Narrationen ähnelten, wie ZILLING herausarbeitet. ${ }^{36}$

Andererseits kann in einer Welt, in der das Christentum immer mehr dominierte, die Hervorhebung der Mythen bei Nicht-Christen Ausdruck eines offensiven Traditionsbewusstseins sein, das sich gerade dem Christentum verweigerte und auf die Unterscheidung davon wert legte. Philosophisch überhöht werden konnte dies, indem man wie in der neuplatonischen Interpretation die Mythen als legitime Fiktion verstand. Die religiöse Aufladung des Mythos durch manche christliche Polemiker wurde von Heiden übernommen; doch blieb dies eine Erscheinung, die wohl nur eine Minderheit betraf. ${ }^{37}$

Wenn der Umgang des Christentums mit Themen der Mythologie keineswegs einheitlich war, so ergibt sich daraus eine naheliegende Schlussfolgerung: Es war nicht nur die Adaptabilität des Mythos, die sein Überleben sicherte, sondern auch die Vielfalt des Christentums, die dazu beitrug. So wie es verschiedene Christentümer gab, gab es auch verschiedene Umgangsweisen mit dem Mythos. Man wird zudem stärker als früher die Gemeinsamkeiten von Christen und Heiden hervorheben, so die Allgegenwart eines Platonismus in den gebildeten Milieus oder die strukturellen Ähnlichkeiten zwischen heidnischen und christlichen Narrationen, auf die verschiedene Beiträge hingewiesen haben. Möglicherweise wäre es aufschlussreich, in Hinblick auf das Verhältnis zum Mythos einen Vergleich zwischen der griechisch-römischen und denjenigen christlichen Kulturen durchzuführen, deren Schriftlichkeit nicht auf dem klassischen Kanon aufbaute, namentlich der syrischen, aber das kann hier nicht geschehen.

Was die zeitliche Gliederung angeht, so bestätigt sich das von Manfred Fuhrmann entwickelte Phasenmodell, allerdings lediglich für den Bereich der Literatur. Hier verfestigt sich der Eindruck, dass im 5. Jahrhundert der Mythos eine stärkere Präsenz hatte als im 4. Jahrhundert, besonders deutlich in der Gattung des Epos. Diese Entwicklung mag insofern paradox erscheinen, als um die Wende zum 5. Jahrhundert das Christentum Dominanz entwickelte; doch scheint in eben dieser Zeit paideia wieder an Bedeutung für die Statusdistinktion der weitgehend sich zum Christentum bekennenden Eliten gewonnen zu haben, so dass mythologische Themen nicht religiös, sondern intellektuell distinktiv waren. Es mochte angesichts der Überlegenheit des Christentums auch schwierig anmuten, den Mythos als gefährlich zu beschreiben. In dieser Zeit genügte es in der Elitenkommunikation offenbar, andeutungsweise Hin-

36 In diesem Band Zilling, jüngst Anna Ferrari, La rilettura cristiana dei miti pagani, AnTard 19 (2011), $209-222$.

37 Zur Übersetzung von Fremdwahrnehmung in Selbstwahrnehmung im spätantiken Heidentum Hartmut Leppin, Zum Wandel des spätantiken Heidentums, Millennium 1 (2004), 59 - 81. 
weise auf Mythen zu geben, deren Verständnis man voraussetzen durfte; SCHMITZER betont, dass dies auf spätere Renaissancen vorauswies.

Im Epos des 6. Jahrhunderts scheinen mythologische Motive hingegen einen Rückgang erlebt zu haben. ${ }^{38}$ In dieser Zeit, der Epoche des totalisierenden christlichen Diskurses, ändert sich anscheinend auch sonst vieles: Die Zahl mythologischer Darstellungen verringerte sich offenbar nicht nur im Epos, sondern auch auf Textilien, also in einer ganz anderen Welt, aber erneut gilt: Die Entwicklung verläuft nicht linear. Denn unter Herakleios gewannen, wie MEIER verdeutlicht, mythologische Elemente eine ganz neue Bedeutung für die kaiserliche Selbstdarstellung.

Daraus ergibt sich eines ganz deutlich: Christentum und die Aneignung mythologischer Themen schließen sich nicht aus. Ein Nebeneinander war, wie RüPKE zeigt, schon im 4. Jahrhundert möglich, und es blieb auch so. Welcher Bildsprache man sich bediente, hing ab von der Funktion des Mediums und der Zielgruppe, aber gewiss auch von persönlichen Präferenzen von Schöpfern oder Auftraggebern. Vieles bleibt im Dunkeln, aber die Vorstellung einer fundamentalen Opposition von Christentum und Mythos setzt eine religiöse Eindeutigkeit des Mythos voraus, die es in den Augen vieler Zeitgenossen offenbar nicht besaß.

Doch wird man hier weitere Differenzierungen anbringen müssen: Bedeutung behielten gewiss auch regionale Unterschiede. Orte pflegten auf ihre jeweilige Art mythische Traditionen, wie ANDo für Rom und BASSETT für Konstantinopel verdeutlicht. Man könnte dann auch über die Frage einer Sonderstellung Ägyptens reflektieren, wie sie aus KRISTENSENs Beitrag erhellen mag. Es wäre also zu überlegen, ob das Nebeneinander christlicher und nicht-christlicher Motive, das man hier feststellt, eine Besonderheit dieser Landschaft war oder aber den besonderen Erhaltungsbedingungen in Hinblick auf Textilien in der Gegend zu verdanken ist. ${ }^{39}$

Blickt man weiter nach Westen, so spricht die umfänglich erhaltene Dichtung dafür, dass das Vandalenreich mit seiner blühenden lateinischen Literatur ebenfalls eine Sonderstellung einnahm; erinnert sei an Namen wie den des Poeten Dracontius und den des im Mittelalter außerordentlich einflussreichen Mythographen Fulgentius, der vermutlich in diesen Kontext gehört. ${ }^{40}$ Überdies wird man soziale Unterschiede in

38 Claudia Schindler, Per carmina laudes. Untersuchungen zur spätantiken Verspanegyrik von Claudian bis Coripp (Beiträge zur Altertumskunde 253), Berlin/New York 2009.

39 Zum Nebeneinander von Christlichem und Heidnischem in der ägyptischen Literatur Leslie S. B. MacCoull, Dioscorus of Aphrodito. His Work and his World (The Transformation of the Classical Heritage 16), Berkeley 1988, besonders plastisch das Gedicht H5, 9-31. - Regionale Unterschiede bei der Dekoration von Öllampen zwischen Nordafrika und Athen stellt Lund (s. Anm. 24), 205 f. fest.

40 Roswitha Simons, Dracontius und der Mythos. Christliche Weltsicht und pagane Kultur in der ausgehenden Spätantike (Beiträge zur Altertumskunde 186), München u.a. 2005, insbes.95-114, zeigt unterschiedliche Formen des Umgangs mit Mythen bei einer grundsätzlichen, oft aggressiven Ablehnung; vgl. Doreen Selent, Allegorische Mythenerklärung in der Spätantike. Wege zum Werk des Dracontius (Litora classica 2), Rahden/Westf. 2011, die den Begriff der Allegorie für Dracontius fruchtbar macht und für die Neukontextualisierung der Mythen den Begriff der „Transmythologisierung“ vorschlägt (zu Fulgentius 49-55); John Chance, Medieval Mythography. From Roman North Africa to the 
den Blick nehmen müssen, die über die Feststellung der Bedeutung von Mythen für die soziale Distinktion der Eliten hinausgehen, denn auch in anderen Schichten scheint der Mythos seine Präsenz bewahrt zu haben: Das Beispiel der Textilien spricht dafür, dass auch middle classes sich von Mythen affizieren ließen. Zudem ist zu berücksichtigen, inwieweit eine mythisch inspirierte Bildsprache oder Narration auch außerhalb der Eliten oral weiterleben konnte, etwa in Zaubersprüchen und Beschwörungsformeln. ${ }^{41}$

Doch bei allen Differenzierungen: In den klassischen Mythen war eine symbolische Tradition gespeichert, die sich immer neuen Aneignungsprozessen erschloss. Der Mythos insgesamt ließ sich von den Christen nicht fangen, mochten auch Teile unter Verschluss geraten. In der Renaissance öffneten sich ganz andere Türen, doch keine führte zurück in die alte, klassische Welt des Mythos, sondern zu neuen Formen der Aneignung, die bis heute kein Ende gefunden haben.

School of Chartres, A.D. 433-1177, 2 Bde., Gainesville/Flor. 2001 erörtert eindringlich die vielfältigen Formen des Umgangs mit dem Mythos in lateinischer Spätantike und Mittelalter; s. Bd. 1, 95-128 zu Fulgentius.

41 Etwa David Frankfurter, The Laments of Horus in Coptic. Myth, Folklore and Syncretism in Late Antique Egypt, in: Ueli Dill/Christine Walde (Hg.), Antike Mythen. Medien, Transformationen und Konstruktionen. Fritz Graf zum 65. Geburtstag, Berlin/New York 2009, 229-247. 